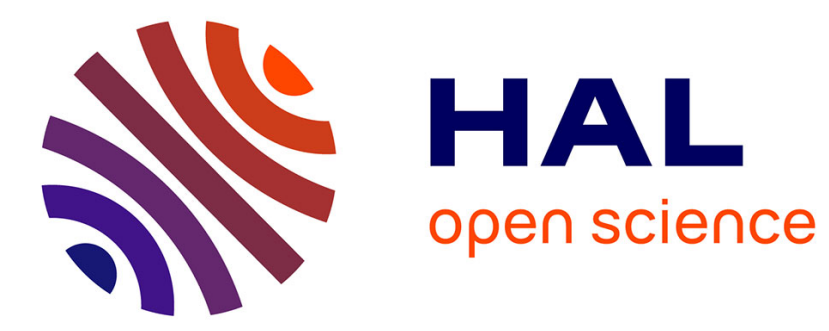

\title{
Estimating the poroelastic properties of cracked materials
}

Laurent Charpin, Alain Ehrlacher

\section{To cite this version:}

Laurent Charpin, Alain Ehrlacher. Estimating the poroelastic properties of cracked materials. Acta Mechanica, 2014, pp.1-19. 10.1007/s00707-013-1082-0 . hal-00983341

\section{HAL Id: hal-00983341 \\ https://hal-enpc.archives-ouvertes.fr/hal-00983341}

Submitted on 25 Apr 2014

HAL is a multi-disciplinary open access archive for the deposit and dissemination of scientific research documents, whether they are published or not. The documents may come from teaching and research institutions in France or abroad, or from public or private research centers.
L'archive ouverte pluridisciplinaire HAL, est destinée au dépôt et à la diffusion de documents scientifiques de niveau recherche, publiés ou non, émanant des établissements d'enseignement et de recherche français ou étrangers, des laboratoires publics ou privés. 


\title{
Estimating the poroelastic properties of cracked materials
}

Received: date / Accepted: date

\begin{abstract}
We present a comparative study of the ability of some micromechanics estimates to predict the overall properties of heterogeneous materials. We focus mainly on cracked materials, for which this task is difficult and many estimates fail. We study particularly the Interaction Direct Derivative estimate, proposed by Zheng and Du, which is an approximation of the generalized self-consistent scheme but has the very convenient property to be always explicit. A modified version of this estimate, called Full Range IDD by Zheng and Du, yields good results when comparing all poromechanical coefficients predicted by the estimate to finite element simulations of a $2 d$ cracked material in plane strain, up to crack density factors of 1 for aligned cracks and 0.60 for randomly oriented cracks. The accuracy of finite element computations of the overall moduli is also commented by plotting the convergence of the average of the properties as well as the confidence intervals on these averages.
\end{abstract}

Keywords Micromechanics · Cracked elastic solid · Interaction Direct Derivative · finite element simulation

\section{Introduction}

The field of micromechanics is dedicated to the prediction of the overall properties of composites, starting from information about the volume fraction, morphology, and mechanical properties of the various phases. Numerous estimates for the mechanical properties have been proposed, and some of them have become very classical, such as the dilute scheme (directly derived from Eshelby's solution [1]), the self-consistent scheme [2], the differential scheme [3], and the Mori-Tanaka scheme [4]. These estimate give more or less good results depending on the volume fractions of the phases and the morphology types considered. For example, Mory-Tanaka's estimate is considered to be particularly adapted for matrix/inclusion morphologies.

Part from these very classical estimates, more refined estimates exist. The general self-consistent estimate seems the most complete [5]. It is, as the mentioned estimates, based on a simple auxiliary problem on which the localization tensors on the inclusionary phases of the real problem are estimated. It has the advantage of taking the distribution of the inclusions into account, in addition to their shape. This is done through an ellipsoid containing the matrix material, embedding the inclusion, and itself embedded into the homogenized material which is sought for. However, it has a closed-form solution in only few cases: isotropically distributed spherical inclusions (this solution was given by Christensen and Lo [6]) and some cases of cracked medium [7]. For other cases, it is necessary to use approximations (such as the one proposed by Hori and Nemat-Nasser in the same article [5]), or to simplify the

L. Charpin and A. Ehrlacher

Université Paris Est, Laboratoire Navier, École des Ponts ParisTech, 6-8 av Blaise Pascal, Cité Descartes, Champs-sur-Marne, 77455 Marne-la-Vallée Cedex 2, France

E-mail: laurent.charpin@enpc.fr 
problem (as is done in the two-phase model, where the matrix material also occupies the infinite medium embedding the matrix ellipsoid [8]). It is also possible to evaluate the general self-consistent solution numerically, as is well known and will be illustrated in this article.

More recently, two improvements have been achieved. First, Ponte-Castañeda and Willis showed precisely the role of the distribution of the inclusions in the framework of Hashin-Strickman principle $[9,10]$. This work, which can be used to provide bounds or an estimate for the elasticity tensor, clarifies the underlying microstructures more or less explicitly considered when establishing the classical estimates. A major point is that the distribution functions of pairs of inclusion families need to be symmetric. In most estimates which consider the distribution of the inclusions, only one distribution is chosen for each inclusion, neglecting this point. However, when the various inclusion families have different distributions, the estimate is not explicit and difficult to use. Second, Zheng and Du proposed an estimate based on the generalized self-consistent model called the Interaction Direct Derivative estimate, which has the advantage of being fully explicit in all cases, even with multiple inclusion families, each having a different space distribution [11].

Concerning the accuracy of these estimates, the classical estimates give good results with simple morphologies such as discs in $2 d$ or spheres in $3 d[12,13,14]$. However, when moving to cracked microstructures, it is much more difficult to predict the overall properties of a Representative Elementary Volume of the material. There has been a number of studies in this direction in $2 d$ (see $[15,7]$ ), but it seems some improvements remain possible. We won't tackle the problem of $3 d$ cracked structures which requires large computational tools for a rigorous treatment, but we will attempt to assess the efficiency of Interaction Direct Derivative estimate of Zheng and Du for cracked structures in $2 d$. We will study its efficiency by comparison to finite element computations, using monodisperse cracks, to the highest crack density factor we were able to reach using a Random Sequential Addition algorithm [16], which was roughly 1 in the case of aligned cracks and 0.6 in the case of randomly oriented cracks. Of course this limit could be improved by using multiple crack sizes, but we won't discuss this point in this article.

We believe that our article brings interesting information in addition to Shen and Li [7] where the case of parallel and aligned cracks in $2 d$ is also dealt with. First, since we deal with cracks, we think that it is important to foresee the application to poromechanics of the homogenization procedure, by computing and displaying the overall poromechanical coefficients such as the Biot coefficient and Biot modulus [17], which we do systematically when comparing the simulations to the estimates. Second, we use the $I D D$ estimate which is very versatile, unlike the solutions developed in some particular cases of aligned or randomly oriented cracks (see Shen and Li [7]), and unlike the differential scheme and the self-consistent scheme in complicated situations. Third, we take some time to comment the error made in the finite element simulations, so that the reader knows exactly to what extent our results can be trusted.

The authors used the $I D D$ estimate for the prediction of degradation of concrete submitted to Alkali-Silica Reaction. This estimate was used to improved a linear elastic fracture mechanical model proposed in [18]. Therefore, more details about the comparison of the IDD estimate and finite element simulations can be found in the author's Ph.D. thesis, as well as applications to Alkali-SIlica Reaction [19].

The article is organized as follows. First, we give a brief explanation of the variational formulations as well as the boundary conditions we use in the finite element code. Second, we describe the procedure of determination of the overall properties. We then present the various microstructures that we study in this article, and explain how we describe the error made in the finite element simulations. We then move to the micromechanics by recalling the expressions and main features of the estimates we use, and finally compare them to the results obtained in the simulations, showing that the IDD estimate is well suited to compute the overall properties of cracked materials.

\section{Plane strain variational formulation}

All computations were done in FreeFem++ [20]. In this software the user implements its own variational formulation, which will be shortly presented here. We decided two work in $2 d$, thinking that in the view of assessing the efficiency of micromechanics estimates, we could obtain first results. Of course to be able to test all possibilities in terms of orientation of inclusions, for example, $3 d$ simulations would be preferable. We will also present shortly the boundary conditions that were used for the computations. 
Our variational formulation is written starting from the virtual work principles, as done classically, in a static case [21]. We call $\Omega$ the continuum solid of local tensor of elasticity $\mathbb{C}(\underline{x})$, and boundary $\partial \Omega$. The external boundary is split between the external boundary $\partial \Omega_{\text {ext }}$ and an internal boundary $\partial \Omega_{\text {int }}$ which separates the solid from pores embedded in the solid. The internal boundary is submitted to pressure loadings, while the external boundary can be submitted to imposed tractions $\underline{T}^{D}$ and imposed displacements $\underline{u}^{D}$.

Since we are in plane strain, the displacement is assumed to be zero in direction 3 and hence, the related components of the strain are also zero:

$$
\varepsilon_{13}=\varepsilon_{23}=\varepsilon_{33}=0
$$

Let us quickly describe the boundary conditions we use.

\subsection{Imposed average strain}

The boundary conditions we call imposed strain are such that the imposed displacement on the external boundary is $\underline{u}^{D}(\underline{x})=\underline{E} \cdot \underline{x}, \forall \underline{x} \in \partial \Omega_{\text {ext }}$, where $\underline{E}$ is a second order, symmetric, plane strain tensor. Calling $\mathcal{C}\left(\underline{u}^{D}\right)$ the space of displacements admissible with $\underline{u}^{D}$, and assuming a pressure $p$ exerts on the internal boundary, the variational formulation is (calling $\underline{\underline{\varepsilon}}$ the strain tensor):

$$
\text { Find } \underline{u} \in \mathcal{C}\left(\underline{u}^{D}\right) \text { such that } \forall \underline{w} \in \mathcal{C}(\underline{0}), \int_{\Omega} \underline{\varepsilon}[\underline{u}]: \mathbb{C}: \underline{\underline{\varepsilon}}[\underline{w}] d V+\int_{\partial \Omega_{i n t}} p \underline{n} \cdot \underline{w} d S=0
$$

\subsection{Imposed average stress}

The boundary conditions we call imposed stress are such that the tractions on the external boundary are equal to $\underline{T}^{D}(\underline{x})=\underline{\Sigma} \cdot \underline{n}, \forall \underline{x} \in \partial \Omega_{\text {ext }}$ where $\underline{\Sigma}$ is a second order symmetric tensor. Let us remark that the average stress is only partly imposed $\overline{\bar{b}}$ ecause we are still in the plane strain framework. Therefore, only the components $\left(\Sigma_{11}, \Sigma_{12}, \Sigma_{22}\right)$ are imposed. Calling $\mathcal{C}$ the space of admissible plane strain displacements, still considering the pressure $p$ is applied in the pores, the variational formulation is:

Find $\underline{u} \in \mathcal{C}$ such that $\forall \underline{w} \in \mathcal{C}, \int_{\Omega} \underline{\varepsilon}[\underline{u}]: \mathbb{C}: \underline{\underline{\varepsilon}}[\underline{w}] d V+\int_{\partial \Omega_{\text {int }}} p \underline{p} \cdot \underline{w} d S-\int_{\partial \Omega_{\text {ext }}^{T}}(\underline{\underline{\Sigma}} \cdot \underline{n}) \cdot \underline{w} d S=0$

\subsection{Periodic boundary conditions}

The boundary conditions we call periodic are such that the displacement on the external boundary is equal to $\underline{u}=\underline{E} \cdot \underline{x}+\underline{u}^{\prime}, \forall \underline{x} \in \partial \Omega_{\text {ext }}$ where the field $\underline{u}^{\prime}$ is periodic and $\underline{E}$ again is a plane strain second order symmetric tensor. Let us decompose the strain as $\underline{\varepsilon}[\underline{u}]=\underline{\underline{E}}+\underline{\underline{\varepsilon}}\left[\underline{u}^{\prime}\right]$ and call $\mathcal{C}^{\text {per }}$ the space of periodic place strain displacement fields. The variational formulation is:

Find $\underline{u}^{\prime} \in \mathcal{C}^{p e r}$ such that $\left.\forall \underline{w} \in \mathcal{C}^{p e r}, \int_{\Omega} \underline{\underline{\varepsilon}}\left[\underline{u}^{\prime}\right]: \mathbb{C}: \underline{\underline{\varepsilon}} \underline{w}\right] d V+\int_{\Omega} \underline{\underline{E}}: \mathbb{C}: \underline{\varepsilon}[\underline{w}] d V+\int_{\partial \Omega_{\text {int }}} p \underline{p} \cdot \underline{w} d S=0$

These various types of boundary conditions in place strain allow us testing our microstructure to determine their average properties. Let us see how we determine these properties from the numerical results. 


\section{Determination of the average properties}

First, we need to be able to compute strain and stress averages. For a quantity $f$ and a domain $V$, the volume average of $g$ over $V$ is $\langle g\rangle_{V}=\int_{V} g d V$. Let us remind these quantities can be computed as surface integral which is sometimes convenient. For example for the average strain:

$$
<\underline{\underline{\varepsilon}}>_{\Omega}=\frac{1}{\Omega} \int_{\partial \Omega_{e x t}} \underline{u} \otimes^{s} \underline{n} d S
$$

Where $\otimes^{s}$ is the symmetrized tensorial product. Let us also recall how the pressure appears in the average stress, assuming the volume fraction of pores is $f=\Omega^{p} / \Omega$ :

$$
<\underline{\underline{\sigma}}>_{\Omega}=-f p \underline{\underline{\mathbb{1}}}+\frac{1}{\Omega} \int_{\Omega \backslash \Omega^{p}} \underline{\underline{\sigma}} d V
$$

\subsection{Overall constitutive law}

Knowing the local elastic behaviour of the solid, and for various imposed macroscopic strains and pressures in the pores, we want to be able to write the macroscopic constitutive law of our porous medium. Following the presentation of Dormieux et al. [22], assuming in this work that there is only one pore family (which does not mean that they are all identical, but that we study their volume changes and the effect of an applied pressure in them as a whole), we can write the macroscopic constitutive law linking the macroscopic stress $\underline{\underline{\Sigma}}$ and the porosity variation of the porous phase $(\phi-f)$ to the average strain $\underline{\underline{E}}$ and the pressure in the pores $p$ :

$$
\left\{\begin{array}{l}
\underline{\underline{\underline{E}}}=\mathbb{C}^{\text {hom }}: \underline{\underline{E}-p \underline{\underline{B}}} \\
\bar{\phi}-f=\underline{\underline{B}}: \underline{\underline{\underline{M}}}+p
\end{array}\right.
$$

Where $\mathbb{C}^{\text {hom }}$ is the homogenised stiffness tensor, $\underline{\underline{B}}$ is the Biot coefficient, while we call $M$ the Biot compliance, inverse of the usual Biot modulus $N$ as presented in Dormieux et al. [22]. These homogenized properties can all be computed using the solution under imposed strain alone, without solving the problem with a pressure in the pores (this is specific to the case of equation 7 where all cavities are considered together). The strain localisation tensor is a fourth order tensor with the minor symmetry $\mathbb{A}$ (such that when a macroscopic strain $\underline{\underline{E}}$ is imposed, the local strain writes, by virtue of the linearity of the problem, $\mathbb{A}(\underline{x}): \underline{\underline{E}})$. Assuming that the solid matrix is homogeneous of tensor elasticity $\mathbb{C}^{0}$ and compliance $\mathbb{S}^{0}$, the microporomechanics theory then allows writing the homogenized poroelastic properties as:

$$
\left\{\begin{array}{l}
\mathbb{C}^{h o m}=(1-f) \mathbb{C}^{0}:<\mathbb{A}>_{\Omega \backslash \Omega^{p}} \\
\underline{\bar{B}}=f \underline{\underline{\mathbb{1}}}:<\mathbb{A}>_{\Omega^{p}} \\
\bar{M}=\underline{\underline{\mathbb{1}}}: \mathbb{S}^{0}:(\underline{\underline{B}}-f \underline{\underline{1}})
\end{array}\right.
$$

\subsection{Precise determination of the overall properties from strain and stress averages}

In this section we provide the expressions we used for the determination of the properties, in the general case where the overall medium is orthotropic. Some of these expressions simplify if the medium is transversely isotropic but we won't detail this case. Let us mention that we use the Voigt notation, with the convention that all symmetric second order tensors (particularity the stress, strain, and Biot coefficient) are written:

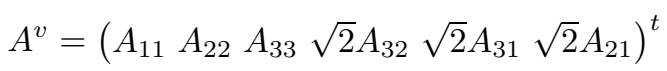


The fourth order are written accordingly:

$$
\mathbb{D}^{v}=\left(\begin{array}{cccccc}
D_{1111} & D_{1122} & D_{1133} & \sqrt{2} D_{1123} & \sqrt{2} D_{1113} & \sqrt{2} D_{1112} \\
D_{2211} & D_{2222} & D_{2233} & \sqrt{2} D_{2223} & \sqrt{2} D_{2213} & \sqrt{2} D_{2212} \\
D_{3311} & D_{3322} & D_{3333} & \sqrt{2} D_{3323} & \sqrt{2} D_{3313} & \sqrt{2} D_{3312} \\
\sqrt{2} D_{2311} & \sqrt{2} D_{2322} & \sqrt{2} D_{2333} & 2 D_{2323} & 2 D_{2313} & 2 D_{2312} \\
\sqrt{2} D_{1311} & \sqrt{2} D_{1322} & \sqrt{2} D_{1333} & 2 D_{1323} & 2 D_{1313} & 2 D_{1312} \\
\sqrt{2} D_{1211} & \sqrt{2} D_{1222} & \sqrt{2} D_{1233} & 2 D_{1223} & 2 D_{1213} & 2 D_{1212}
\end{array}\right)
$$

Second, let us remind that we work in plane strain to reproduce a $3 d$ material which would be invariant and infinitely extended in direction 3. The matrix is of Young's modulus and Poisson's ratio $(E, \nu)$. This analogy to the infinite medium case is useful when coefficients relative to direction 3 are needed. It can be shown that regardless of the shape of the voids, at volume fraction $f$, the Young's modulus and Poisson's ratio in direction 3 write ([23], p.85):

$$
\begin{aligned}
E_{3} & =(1-f) E \\
\nu_{32} & =\nu_{31}=\nu
\end{aligned}
$$

While the Biot coefficient in direction 3 writes:

$$
B_{33}=f\left[1+\nu\left(A_{11}^{v}+A_{12}^{v}+A_{21}^{v}+A_{22}^{v}\right)\right]
$$

Let us then show, as an example, how we determine the poromechanical coefficients in the case where the macroscopic strain is prescribed (imposed strain or periodic boundary conditions). Three simulations are performed (we don't use equation 8, but determine the coefficients directly from equation 7). During the first one, the following macroscopic strain is imposed:

$$
\left(E^{I}\right)^{v}=\left(\begin{array}{llllll}
1 & 0 & 0 & 0 & 0 & 0
\end{array}\right)^{t}
$$

And average stresses $\Sigma_{11}^{I}, \Sigma_{22}^{I}$ et $\Sigma_{12}^{I}$ are measured. Second, the macroscopic strain

$$
\left(E^{I I}\right)^{v}=\left(\begin{array}{llllll}
0 & 1 & 0 & 0 & 0 & 1
\end{array}\right)^{t}
$$

is imposed, while average stresses $\Sigma_{11}^{I I}, \Sigma_{22}^{I I}$ et $\Sigma_{12}^{I I}$ are measured. We can then write the following expressions for the elastic properties:

$$
\begin{gathered}
E_{1}=\frac{\left(\frac{\Sigma_{11}^{I}}{\Sigma_{22}^{I}}\right)^{2}-\left(\frac{\Sigma_{11}^{I I}}{\sum_{22}^{I I}}\right)^{2}}{\frac{1}{\left(\Sigma_{22}^{I}\right)^{2}}\left[\Sigma_{11}^{I}+\frac{\nu^{2}}{E_{3}}\left(\left(\Sigma_{11}^{I}\right)^{2}-\left(\Sigma_{22}^{I}\right)^{2}\right)\right]+\frac{1}{\left(\Sigma_{22}^{I I}\right)^{2}}\left[\Sigma_{22}^{I I}+\frac{\nu^{2}}{E_{3}}\left(\left(\Sigma_{22}^{I I}\right)^{2}-\left(\Sigma_{11}^{I I}\right)^{2}\right)\right]} \\
E_{2}=\frac{\left(\frac{\Sigma_{22}^{I I}}{\Sigma_{11}^{I I}}\right)^{2}-\left(\frac{\Sigma_{22}^{I}}{\Sigma_{11}^{I}}\right)^{2}}{\frac{1}{\left(\Sigma_{11}^{I}\right)^{2}}\left[\Sigma_{11}^{I}+\frac{\nu^{2}}{E_{3}}\left(\left(\Sigma_{11}^{I}\right)^{2}-\left(\Sigma_{22}^{I}\right)^{2}\right)\right]+\frac{1}{\left(\Sigma_{11}^{I I}\right)^{2}}\left[\Sigma_{22}^{I I}+\frac{\nu^{2}}{E_{3}}\left(\left(\Sigma_{22}^{I I}\right)^{2}-\left(\Sigma_{11}^{I I}\right)^{2}\right)\right]} \\
\nu_{12}=\frac{E_{1}}{\Sigma_{22}^{I I}}\left[\frac{\Sigma_{11}^{I I}}{E_{1}}-\frac{\nu^{2}}{E_{3}}\left(\Sigma_{11}^{I I}+\Sigma_{22}^{I I}\right)\right] ; \mu_{12}=\frac{\Sigma_{12}^{I I}}{2}
\end{gathered}
$$

Concerning the Biot compliance and coefficient, they are determined imposing zero average strain and a unit pressure in the pores. Using this solution, we then have by definition (see equation 7):

$$
\begin{gathered}
B_{11}=-<\sigma_{11}>_{\Omega} \\
B_{22}=-<\sigma_{22}>_{\Omega} \\
M=\phi-f=f<\operatorname{tr} \underline{\underline{\varepsilon}}>_{\Omega^{p}}
\end{gathered}
$$

The case of imposed stresses is dealt with similarly, but the expressions are not detailed here. 


\section{Studied microstructures}

Let us now describe the type of microsctructures whose overall properties we will study. Let us first talk about the generation of the random microstructures, and then describe the periodic cells we used.

4.1 Random microstuctures built by Random Sequential Addition

This process allows creating matrix/inclusion morphologies easily [16,15,24,13]. We show an example and monodisperse spheres on Fig. 1. Let us briefly sum up the procedure. First, a domain size, inclusion

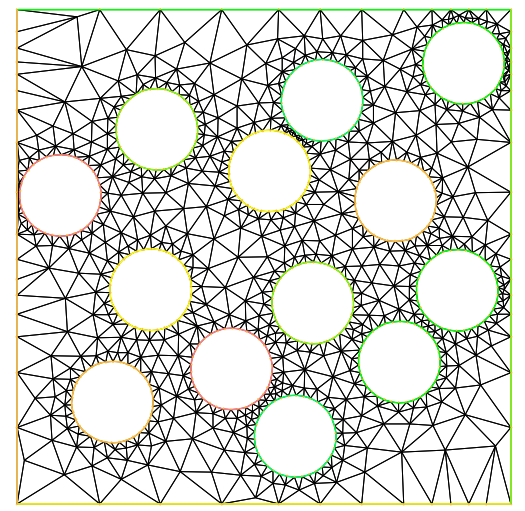

Fig. 1 One realisation of a microstructure with isotropically distributed circular pores

size and volume fraction $f$ are chosen. Then the coordinates of the center of the particle are randomly generated (and, for example, its orientation if necessary). If the particle has no intersection with the external boundary of the domain nor any previously added particle, it is kept. This operation is then repeated until the desired volume fraction is reached, which is more accurate if there is a large number of inclusions.

The first difficulty of the method is that for particle shapes other than aligned ellipsoids, testing the intersection of two particles can be difficult and can require the discretisation of the boundary of the particle. Second, even if for discs in $2 d$ the maximal density is roughly 0.9 , this method, for monodisperse inclusion sizes, creates empty spaces between particles that are too small to be filled by a new particle. The density is therefore limited, for spheres, to roughly 0.5. Finally, for meshing reasons, the particles cannot touch each other. The small exclusion distance reduced even more the density we can reach.

\subsection{Periodic microstructures}

Periodic simulations are convenient and easy to implement. They are based on the choice of a periodic pattern. In our case, for spheres, we followed the choice of Zheng and Du [11], placing the centers of the spheres on a hexagonal pattern. This allows attaining, in $2 d$, optimal densities for monodisperse spheres. In the case of aligned ellipsoids, there are two interesting possible choices. The first possibility is to keep the ellipsoids centered on the same patterns as the spheres, representing an isotropic space distribution of the ellipsoids, but then the volume fraction is geometrically very limited. Another idea is to flatten the hexagonal pattern on which the ellipsoids are placed by the same factor as the aspect ratio of the ellipsoids. An example of obtained pattern in this case is shown on Fig. 2. In this case we can reach the same high density as in the case of spheres. 


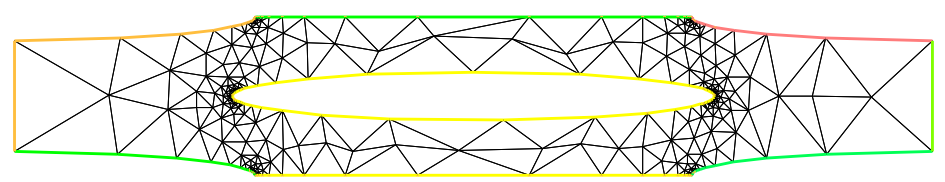

Fig. 2 Periodic cell, inclusion and ditribution of aspect ratio 0.1

\subsection{Remarks concerning the accuracy of the results}

Concerning the size of the elements of the meshes used for the computation, an automatic mesh refinement procedure based on the geometrical error in the representation of the microstructure was used, as well as a limitation of the elongation of the elements. The results are converged with respect to the mesh size.

Another issue is the fact that since our inclusions don't intersect the external boundary of the domain, the inclusion density is lower close to the boundary. However, since the convergence of the poromechanical properties is verified with respect to the ratio between the inclusion size and domain size, the final values which are used for the comparisons with the estimates are not affected by this phenomenon.

\section{Determination of the REV size in a simple case, with Kanit's approach}

To obtain representative values of the homogenized properties thanks to the numerical simulations, we must use domains which are large enough compared to the inclusion sizes. The convergence of these properties with respect to the domain size can be checked for the different types of boundary conditions.

Let us first recall that the obtained tensors of elasticity using our simple boundary conditions always verify the following relation for any second order symmetric tensor $\underline{\underline{E}}$

$$
\underline{\underline{E}}: \mathbb{C}_{\Sigma}^{h o m}: \underline{\underline{E}} \leq \underline{\underline{E}}: \mathbb{C}_{\text {general }}^{\text {hom }}: \underline{\underline{E}} \leq \underline{\underline{E}}: \mathbb{C}_{E}^{h o m}: \underline{\underline{E}}
$$

Where $\mathbb{C}_{\Sigma}^{\text {hom }}$ is determined using the imposed stress boundary conditions, $\mathbb{C}_{E}^{\text {hom }}$ is determined using the imposed strain boundary conditions, while $\mathbb{C}_{\text {general }}^{\text {hom }}$ refers to any boundary conditions. This property of the different stiffness tensor that can be determined on the same microstructure is well known [23, $25]$.

As a consequence, the difference between the moduli obtained with imposed stresses or strains also give an insight about the error which is made.

\subsection{Description of the error on the estimation of the mechanical properties}

Considering a domain of calculus of size $L$ and a characteristic size of the inclusions $R$, we study the convergence of the mechanical properties, for the three types of boundary conditions, when $\frac{R}{L} \rightarrow 0$. There has been many studies of this kind, see for example Gusev [26]. Two sources of error appear: first, a bias due to the the lack of representativity of small domains, which cannot be suppressed even with an infinite number of realizations, and which is more or less pronounced depending on the boundary conditions used. Second, the insufficient number of realizations, which lead to imperfect estimation of the average. Following [27] and [28], we compute the average and the variance of the properties we are interested ( say $A_{i}$ ) for $N$ realizations, by using the classical formula for the average $\mu_{N}(A)=\frac{1}{N} \sum_{i=1}^{N} A_{i}$ and for the variance $\sigma_{N}(A)=\frac{1}{N-1} \sum_{i=1}^{N}\left(A_{i}-\mu_{N}(A)\right)^{2}$. Hence we can plot, for each poroelastic quantity, the empirical average and the confidence interval at $95 \%\left(\mu_{N}(A) \pm 1,96 \sqrt{\sigma_{N}(A) / N}\right)$ as a function of the ratio $\frac{R}{L}$. 
5.2 Example: circular inclusions

We study the three types of boundary conditions, for a fixed volume fraction of circular cavities $f$, hence inducing transversely isotropic overall poromechanical properties. We plot the in-plane shear modulus $\mu_{12}$, a plane strain two-dimension bulk modulus we define as:

$$
k^{2 d d p}=\frac{1}{2} \frac{\Sigma_{11}+\Sigma_{22}}{E_{11}+E_{22}}
$$

from the macroscopic strains and stresses, as well as the Biot compliance $M$ and Biot coefficient $B$.
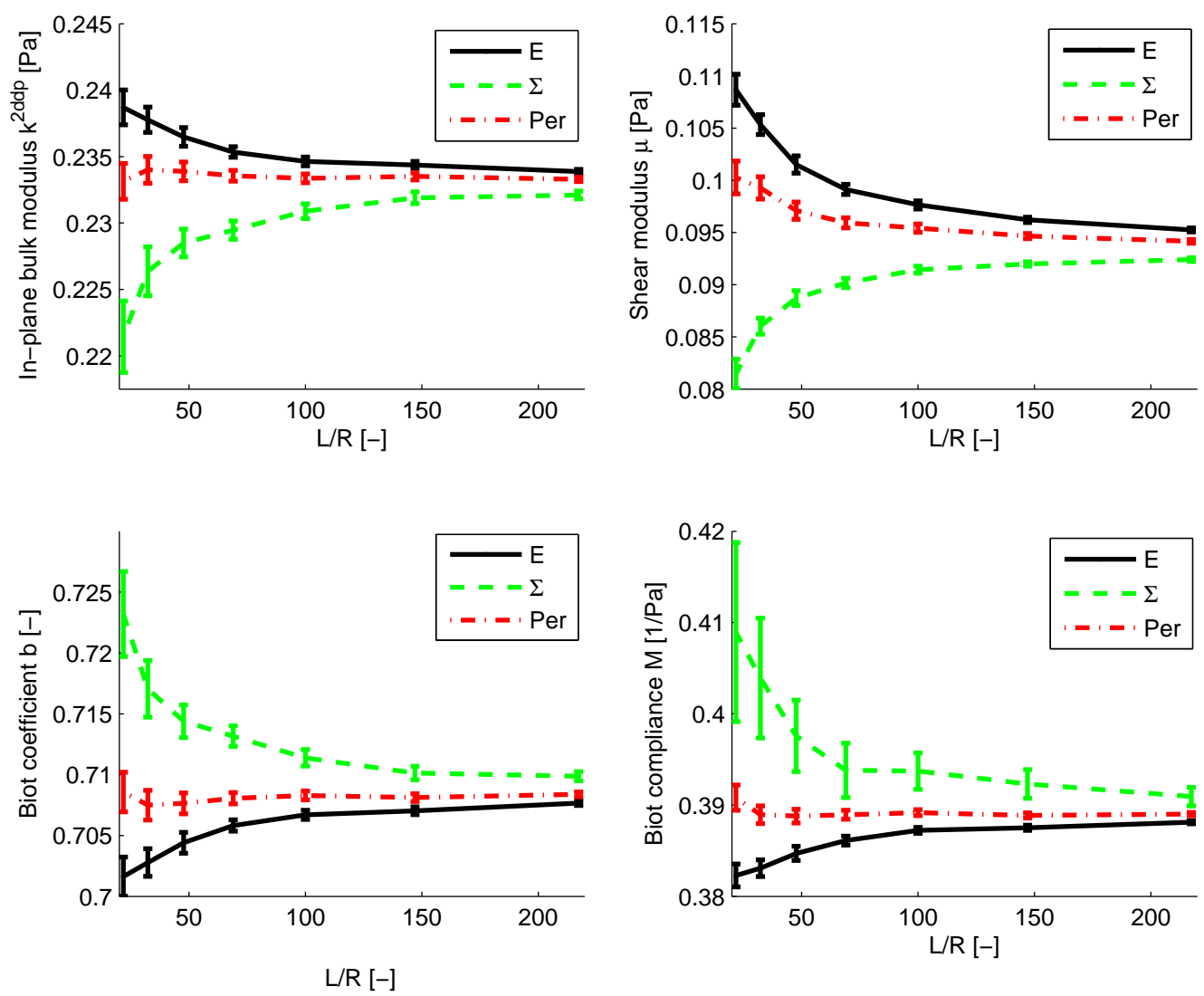

Fig. 3 Evolution of the poromechanical properties with the ratio of the size of cavities to calculus domain $R / L$, for a volume fraction $f=0,4$, Young modulus $E=1 \mathrm{~Pa}$ and Poisson's ratio $\nu=0,25$, for a number of $N=50$ realizations for each case, as well as the $95 \%$ confidence interval

Looking at Fig. 3, we observe that the error bars, representing the uncertainty to which we know the empirical average, get smaller with increasing $R / L$, at fixed number of realizations $N$. The imposed strain and imposed stress solutions are biased, which means that even at large number of realizations, we don't get the converged value of the poromechanical properties. Finally, the periodic boundary conditions show almost no bias, which is encouraging because it means it is possible to use much smaller ratios of the domain size to heterogeneity size to obtain good estimates of the poromechanical properties than with the other boundary conditions. Therefore, from now on, only the periodic boundary conditions will be used.

Let us recall the expressions of the micromechanics estimates we will compare to these finite element results. 


\section{Micromechanics estimates}

For each estimate, we give the expression of the strain localization tensor on the inclusionary phase, as well as the homogenized stiffness tensor, except for the general self-consistent estimate that is not analytical. The classical micromechanics results from Dormieux et al. [22] reminded in equation 8 allow writing the Biot modulus and coefficient easily once the localization tensor is known.

Dilute scheme (dil) The dilute scheme is not shown in the comparisons but is used to build other estimates. Considering $N$ families of ellipsoidal heterogeneities, embedded in a matrix of stiffness tensor $\mathbb{C}^{0}$, each caracterized by their stiffness tensor $\mathbb{C}^{i}$, Hill tensor $\mathbb{P}_{i}^{0}$ and volume fraction $f^{i}$, the dilute localization tensor of family $i$ writes:

$$
\mathbb{A}_{i}^{d i l}=\left[\mathbb{I}+\mathbb{P}_{i}^{0}:\left(\mathbb{C}^{i}-\mathbb{C}^{0}\right)\right]^{-1}
$$

It is the direct application of Eshelby's result [1] and it is used in the construction of all analytical micromechanics estimates. The stiffness tensor is then simply obtained by the following general expression for the stiffness tensor:

$$
\mathbb{C}^{d i l}=\mathbb{C}^{0}+\sum_{i=1}^{N} f^{i}\left(\mathbb{C}^{i}-\mathbb{C}^{0}\right): \mathbb{A}_{i}^{d i l}
$$

Two-phase estimate (2ph) The two phase estimate, when there is only one heterogeneity family, is built by embedding the heterogeneity $\omega$ of stiffness tensor $\mathbb{C}^{i}$ in an infinite elastic medium of same stiffness tensor as the matrix of the composite $\mathbb{C}^{0}[8]$. Then an ellipsoid $D$ embedding the inclusion $\omega$ is defined to represent the spatial distribution of the inclusion rather than its shape, and so that the volume fraction of the inclusions in the real composite $f$ is equal to $\omega / D$. Both ellipsoids are characterized by their Hill tensor. This defines a new localization tensor, linking the average strain on $\omega$ to the average strain on $D$ when a strain is applied at infinity:

$$
\mathbb{A}_{\omega}^{2 p h}=\mathbb{A}_{\omega}^{d i l}:\left[\mathbb{I}-f \mathbb{P}_{D}^{0}:\left(\mathbb{C}^{i}-\mathbb{C}^{0}\right): \mathbb{A}_{\omega}^{d i l}\right]^{-1}
$$

Mori-Tanaka estimate (MT) We then build the famous Mori-Tanaka estimate from the two-phase estimate, by assuming that the atmosphere $D$ is similar to the inclusion $\omega$, which means we use the same Hill tensor, and use equation $25[29,4,30]$. The moduli of cracked materials were originally computed by Zhao et al. [31].

Self-consistent estimate (SC) The self-consistent estimate assumes that the inclusion is embedded in the homogenized material rather than the matrix material [2,32]. Therefore, the self-consistent localization tensor is similar to the dilute localization tensor except for this difference:

$$
\mathbb{A}_{i}^{S C}=\left[\mathbb{I}+\mathbb{P}_{i}^{S C}:\left(\mathbb{C}^{i}-\mathbb{C}^{S C}\right)\right]^{-1}
$$

Where $\mathbb{C}^{S C}$ is unknown, but can be expressed as a function of the localization tensor using equation 24 :

$$
\mathbb{C}^{S C}=\mathbb{C}^{0}+f\left(\mathbb{C}^{i}-\mathbb{C}^{0}\right): \mathbb{A}_{i}^{S C}
$$

Which yields an implicit expression for $\mathbb{C}^{S C}$, which can be difficult to solve when the homogenized medium is not isotropic due to much more complicated expressions of the Hill tensor appearing in the equation. We will only use this estimate in a simple case where we have a closed form solution to this equation. If there is one family of spherical holes in an homogeneous material characterized by a shear modulus and Poisson's ratio $\left(\mu_{0}, \nu_{0}\right)$, then the evolution of the in-plane shear modulus and plane strain two-dimensional bulk modulus as defined in equation 22 write (see Nemat-Nasser and Hori [23], p. 103):

$$
\frac{\mu_{12}^{A C}}{\mu_{0}}=\frac{1-3 f}{1+f\left(1-4 \nu_{0}\right)} ; \frac{k_{2 d d p}^{A C}}{k_{0}^{2 d d p}}=2 \frac{\left(3-4 \nu_{0}\right)\left(1-\nu_{0}\right)(1-3 f)}{3-4 \nu_{0} 1-f\left(1-4 \nu_{0}\right)}
$$


Interaction Direct Derivative estimate (IDD) This estimate is built on the ideas of the generalized self-consistent problem [5], which is recognized to be very good for matrix/inclusion morphologies, but has no closed-form solution except in some simple cases. The auxiliary mechanical problem which is considered to determine the localization tensor for inclusion family $i$ is the the following: inclusion $i$ is embedded in its atmosphere of ellipsoidal shape $D_{i}$ so as to respect the volume fraction $f_{i}=\omega_{i} / D_{i}$ and of stiffness tensor that of the matrix. The atmosphere is itself embedded into an infinite medium which has the mechanical properties we are looking for, as in the self-consistent model. While in Hori and Nemat-Nasser [5], the meaning of the shape of the atmosphere was not clear, later developments such as in Ponte-Castañeda and Willis [9] made it clear that it is related to the spatial distribution of the inclusions, independently from their shape. From this generalized self-consistent set-up, several assumptions are made, which lead to the $I D D$ estimate, as characterized by the localization tensor of inclusion $i$ :

$$
\mathbb{A}_{i}^{I D D}=\mathbb{A}_{i}^{d i l}:\left[\mathbb{I}-\sum_{k} f^{k} \mathbb{P}_{D k}^{0}: \mathbb{A}_{k}^{d i l}\right]^{-1}
$$

This estimate is identical to the two-phase estimate $2 p h$ when there is only one heterogeneity family, and both these estimates also become identical to the Mori-Tanaka $M T$ when the atmosphere is taken similar to the inclusion itself.

It is also identical to the Ponte-Castañeda and Willis estimate in the case where the distribution of the various phases is identical [9]. Therefore, the properties of the $P C W$ estimate explained in [33] can be extended to the $I D D$ estimate when all atmospheres are identical, such as the dynamical basis of the estimate in some simple situations of aligned and randomly oriented cracks. Also, the drawbacks of the $P C W$ estimate underlined in $[34,35,33]$, such as the fact that flat ellipsoids cannot keep an isotropic spatial distribution at high volume fraction, because it leads to a violation of Hashin-Strickman bounds [10], apply to the $I D D$ estimate.

However, the $P C W$ estimate is not explicit when these distributions are different for each inclusionary phase. The $P C W$ estimate is rigorous in the sense that imposing the symmetry of pair-wise distribution functions is required by the Hashin-Strickman framework they use. In the IDD estimate, independent distribution functions can be chosen, which might violate this requirement, but makes the use of the estimate much simpler (as also stated in [14], even if in the cases presented in this paper, these estimates coincide). This estimate won't be used directly here, but we acknowledge that it is of great importance to mention it because it allows understanding the drawbacks of other estimates, for example concerning the symmetry of distribution functions of pairs of families of different inclusions, which can be violated using the $M T$ or $I D D$ estimate [36].

Generalized self-consistent estimate (GSC) This estimate is not in closed form. The GSC set-up explained when presenting the $I D D$ estimate can be dealt with numerically, instead of using approximations such as those of Zheng and Du which yields the IDD estimate or Hori and Nemat-Nasser's [5]. It is solved iteratively: at each step of calculus we have the inclusion material in $\omega$, the matrix in $D \backslash \omega$, and an approximation of the homogenized stiffness tensor outside $D$. A new approximation of the stiffness tensor is determined by imposing a strain at infinity and finding the fourth order tensor that relates the average strains and stress on $D$. At next step, this new stiffness tensor is used for the infinite medium. It has been shown that this algorithm converges, and the limit stiffness tensor is the GSC estimate [37].

\section{Comparisons between the estimates and the finite element simulations}

We now have all elements to test the validity of the estimates in different cases. We will begin with discs, then aligned and randomly oriented ellipses.

\subsection{Discs}

It is the simplest situation, in which the homogenized medium is isotropic, and the distribution of the voids is similar to their shape (see Fig. 1). The study of that type of morphology is classical, whether 
experimentally [12] (in this study the differential scheme performs better than the MTscheme) or numerically [13], where Torquato's third order estimate [38] is preferred to the GSC estimate, while the $M T$ estimate is considered the poorest. Note that we use monodisperse discs, which limits the volume fraction of voids, but was shown for example by Ma (in the case of cracks) not to have a big influence on the mechanical properties [39] and by Kurukuri for spheres [40].

We plot four types of results (see Fig. 4). First, simulation results obtained on the microstructures generated by Random Sequential Addition that we label alea. The periodic boundary conditions were used, and convergence of the properties was checked (see $\S 4.1$ ). Second, simulations on the periodic cell (see $\S 4.2$ ), labelled per. Third, two classical estimates: self-consistent $S C$ and Mori-Tanaka $M T$, which is in this case identical to the $2 p h$ and $I D D$ estimates since there is only one void family and its distribution is similar to its shape. Finally, the $G S C$ which was obtained numerically.

The $S C$ scheme, when compared to the alea, as well as per results, performs poorly. It overestimates the decrease of the mechanical properties. This confirms the general opinion that this estimate is not suitable for matrix/inclusion morphologies, but for percolating morphologies, of the kind we could have obtained by using microstructures generated by an algorithm which allows for the interpenetration of the generated voids. The $M T=I D D=2 p h$ estimate gives good results, as well as the $G S C$ numerical estimate. Interestingly, except for the shear modulus, the estimates are closer to the periodic simulation than to the random simulation.
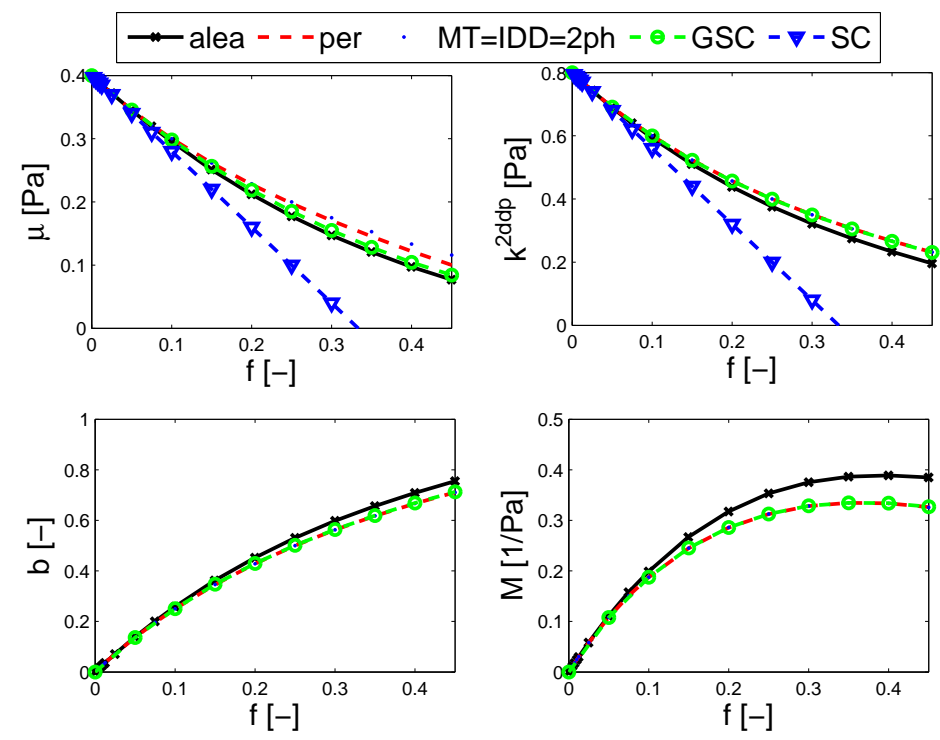

Fig. 4 Evolution of the poromechanical properties as a function of the volume fraction of inclusions of voids shaped as discs, with $E=1 \mathrm{~Pa}$ and $\nu=0.25$, for $N=50$ realizations for each volume fraction for alea. Comparison with classical estimates $(S C$ and $M T=I D D=2 p h)$, computations on a periodic cell per, and the $G S C$ numerical estimate.

\subsection{Flat aligned pores}

In this case the material is no more transversely isotropic as in the case of discs. Its overall properties are described by four coefficients in the plane. We choose two Young's moduli $E_{1}$ and $E_{2}$, the in-plane shear modulus $\mu_{12}$ and the Poisson's ratio $\nu_{12}$. In addition to that, we have two Biot coefficients and the Biot compliance $\left(b_{11}, b_{22}, M\right)$. As was mentioned, the cracks do not intersect with the outer boundary of the domain, as in simulations found in Huang et al. [15]. Huang has concluded in his work that for crack densities up to 0.6 , the $G S C$ estimate is the best, which they only prove it in the case of randomly oriented cracks. However, it seems the computations domains he used were too small compared to the inclusion sizes [7]. It is however encouraging for us, because the IDD estimate is built to approach 
the GSC estimate. In Shen and Li [7], the differential scheme is considered satisfactory up to a crack density of 0.6, while in Yin and Ehrlacher [41] it is shown efficient up to 0.3, but it is not tested above this values.

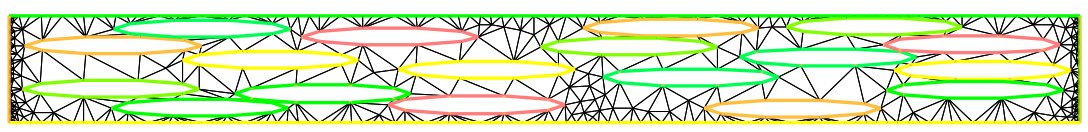

Fig. 5 Flat pores of aspect ratio $\mathrm{X}=1 / 10$. The domain has the same aspect ratio as the pores, so that the error on the estimation of the poromechanical properties is similar in both in-plane directions.

We plot three sorts of curves. First, the results on the microstructures generated by Random Sequential Addition alea. Second, two sorts of simulations on periodic cells, as explained in $\S 4.2$. perEE denotes the simulations in which the distribution of the ellipses is similar to their shape (see Fig. 2), while perEC denotes the simulation in which the ellipses remain distributed isotropically. Finally, two classical estimates: the $2 p h$ estimate, in this case giving the same result as the $I D D$ estimate, in which the distribution of the pores has been chosen as isotropic, and the $M T$ estimate.

The poromechanical properties of this orthotropic medium are plotted on Figs. 6 and 7. Note that the results are displayed as a function of the volume fraction of pores $f$, but when the ellipsoids are of aspect ratio $X$, a link can be made with the classically used crack density factor $\epsilon=f /\left(\frac{4 \pi}{3} X\right)$, as in Budiansky and O'Connell [32].
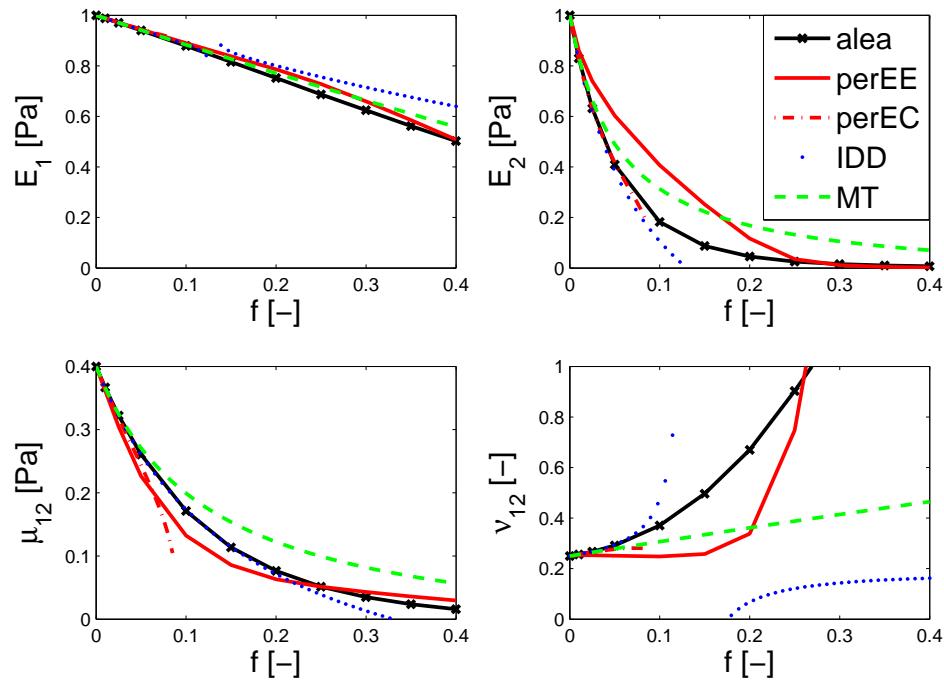

Fig. 6 Evolution of the overall elastic properties as a function of the volume fraction of inclusions of flat pores of aspect ratio $\mathrm{X}=1 / 10$, with $E=1 \mathrm{~Pa}$ and $\nu=0.25$, for $N=50$ realizations for each volume fraction for alea. Comparison with classical estimates $M T$ and $I D D=2 p h$ ), computations on a periodic cell with isotropic distribution perEC and flat distribution perEE. Note that $\mathrm{f}=0.4$ corresponds to a crack density of $\epsilon=0.95$.

First, the $2 p h=I D D$ estimate does not perform well at all. The Young's modulus in direction 2 is estimated as zero at a low volume fraction of 0.13 , which does not match the simulation results. Similarly, the Biot coefficient $b_{2}$ becomes greater than 1 , which is also thermodynamically impossible. The Poisson's ratio is not well estimated either. This failure is related to the fact that when putting the inclusion $\omega$ in the atmosphere $D$ and respecting volume fractions, the first needs to fit in the second, which when the inclusion is flat and the atmosphere is not becomes impossible when increasing the volume fraction, exactly when we reach $f=X$. To solve this problem, we need to work on the choice of the shape of the atmosphere. Compared to this failure of the $2 p h=I D D$ estimates, $M T$ performs better, but the estimate is still poor. 

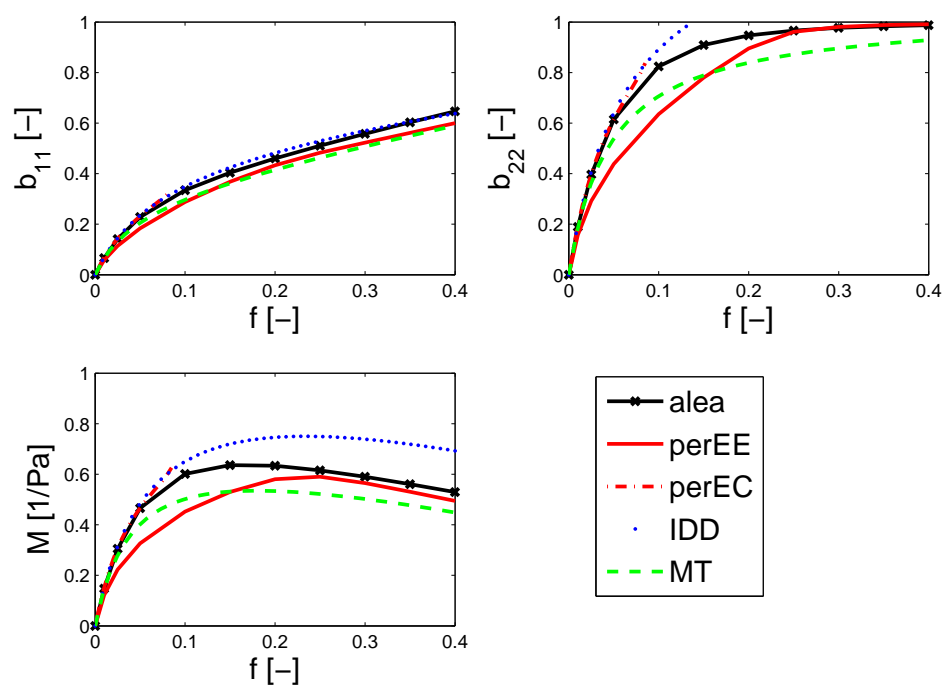

Fig. 7 Evolution of the overall poroelastic properties as a function of the volume fraction of inclusions of flat pores of aspect ratio $\mathrm{X}=1 / 10$, with $E=1 \mathrm{~Pa}$ and $\nu=0.25$, for $N=50$ realizations for each volume fraction for alea. Comparison with classical estimates $M T$ and $I D D=2 p h)$, computations on a periodic cell with isotropic distribution perEC and flat distribution perEE. Note that $\mathrm{f}=0.4$ corresponds to a crack density of $\epsilon=0.95$.

Concerning the simulations on the periodic cells, the two simulations yield very different results. We see that alea is close to perEC at low volume fractions, while getting closer to perEE at large ones. This is related to the process of construction of the microstructure. While generating the inclusions randomly in the domain, no anisotropy is created. However, the rejection of a new void when it intersects with a previously placed void creates anisotropy by making the distribution of the voids in space closer and closer to their shape. This can be measured [24], and tells us what to improve in the $2 p h=I D D$ estimate to avoid the failure occurring when the distribution of flat voids becomes anisotropic at high volume fractions.

As the volume fraction increases, the assumption of isotropic distribution of the inclusions which was made using the $2 p h$ estimate becomes less realistic than the one which is embedded in the $M T$ estimate, in which the distribution of inclusions is similar to their shape. We need to imagine a transition from one regime to the other, inspiring ourselves of the case where the inclusions are placed on the periodic grid (see $[11,9,42]$ ).

Let us come back to the auxiliary problem of the inclusion $\omega$ in the atmosphere $D$, and try to bring a solution to this problem in $2 d$. The inclusion is of aspect ratio $\mathrm{X}$, the atmosphere is of aspect ratio $X^{d}$, and the volume fraction of inclusion is respected $f=\omega / D$. At low $f$, we start with $X^{d}=1$. When we reach $f=X$, the inclusion does not fit in the atmosphere anymore. Notice that the total volume fraction is used, not partial volume fractions according to the orientations. One way of modifying the atmosphere is to choose $X^{d}=X / f$, so that the volume is respected thanks to a flattening of the atmosphere. The atmosphere is then, for each crack, flatten according to this rule and coaxial with the crack. This concept was already proposed by Ponte-Castañeda and Willis [9] in the case of aligned cracks, and Zheng and Du [11] in the case of randomly oriented cracks, under the name Full-Range IDD $(F R I D D)$, and yields an estimate which lies in between the IDD and $M T$ estimates. It is used for example in [43].

Let us see how this estimates performs with flatter ellipses, we will call cracks.

\subsection{Aligned cracks}

A similar analysis is made with flat ellipses of aspect ratio $1 / 100$, which we use to represent cracks in the medium. We represent on Figs. 8 and 9 the results obtained with the $M T, I D D$, and FRIDD estimates. The results obtained with the two first estimates have the same defaults as when we compared them to 
results with $1 / 10$ aspect ratio ellipses. The FRIDD gives an intermediary solution, which is not perfect but preferable to the other estimates since it is thermodynamically correct (for example, the Young's moduli remain positive) and performs better than the $M T$ estimate which generally overestimates the stiffnesses. However, even using this FRIDD estimate, the estimation of the Poisson's ratio is still not satisfactory.
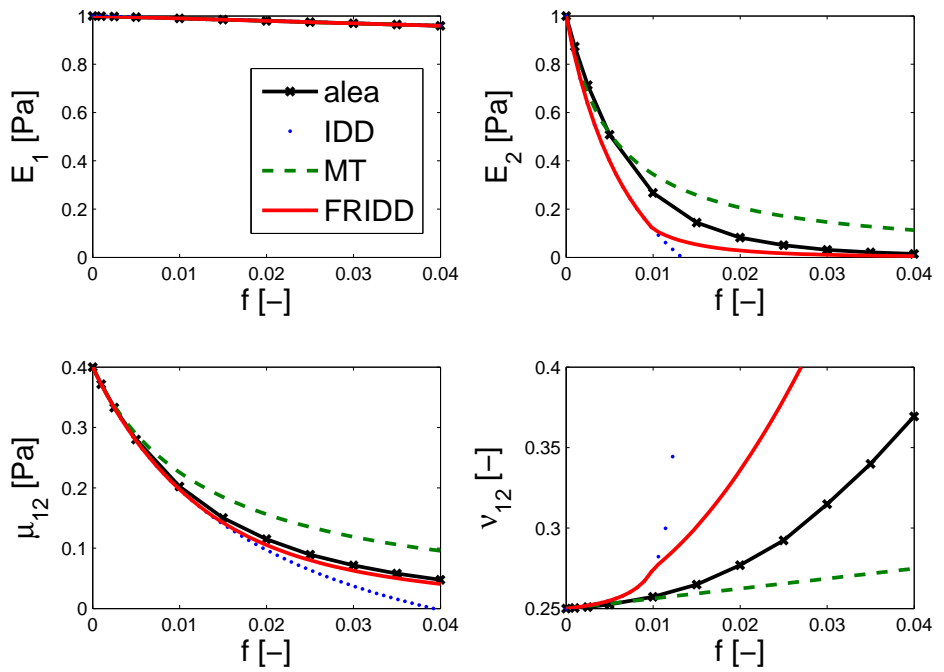

Fig. 8 Cracks of aspect ratio 1/100. Simulations, IDD estimate with circular atmosphere, $M T$ estimate, FRIDD estimate with evolving atmosphere. Elastic properties. Note that $\mathrm{f}=0.04$ corresponds to a crack density of $\epsilon=0.95$.
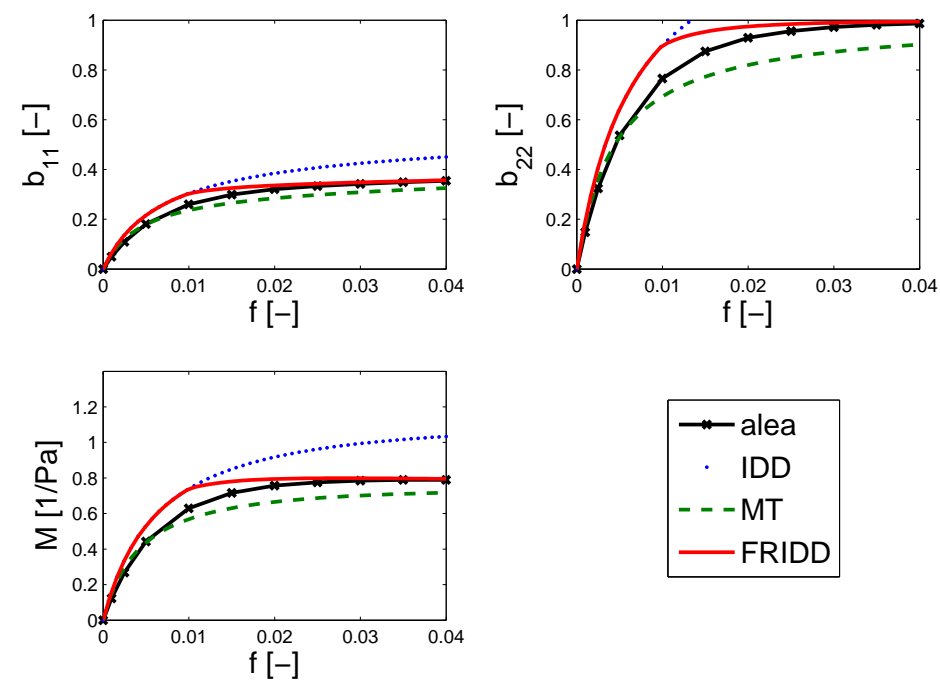

Fig. 9 Cracks of aspect ratio 1/100. Simulations, IDD estimate with circular atmosphere, $M T$ estimate, FRIDD estimate with evolving atmosphere. Poroelastic properties. Note that $\mathrm{f}=0.04$ corresponds to a crack density of $\epsilon=0.95$. 
7.4 Flat pores of random orientation, or in two orthogonal families

In this section we test two possibilities. First, the orientation of the cracks is random, as one can see on Fig. 10. Second, there is two families of orthogonal cracks as on Fig. 11.

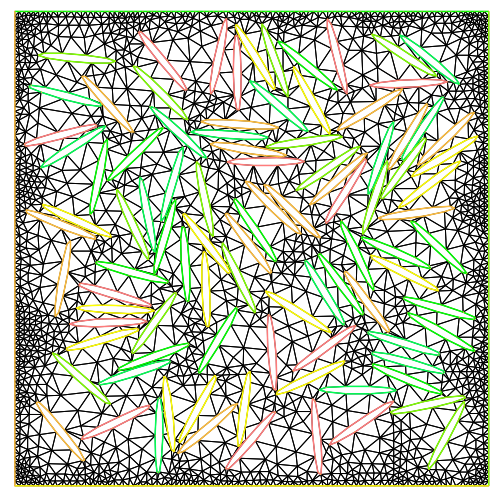

Fig. 10 Microstructure with isotropically oriented cracks

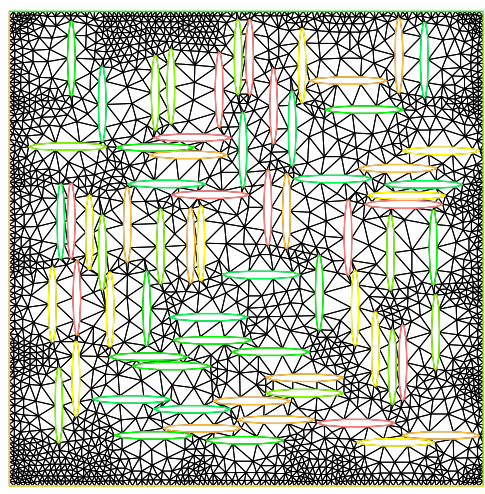

Fig. 11 Microstructure with two orthogonal crack families

When the microstructure contains isotropically oriented cracks, the overall material is transversely isotropic. The accessible volume fractions with the $R S A$ algorithm are smaller than in the case of aligned cracks because some spaces are too small to contain a crack of any orientation but still represent large volume fractions (see Fig. 10). The overall properties of such a microstructure are compared to the three estimates $M T, I D D$, and FRIDD. When the cracks have two orthogonal orientations (Fig. 11), the results are very similar, as can be seen on Fig. 12 .

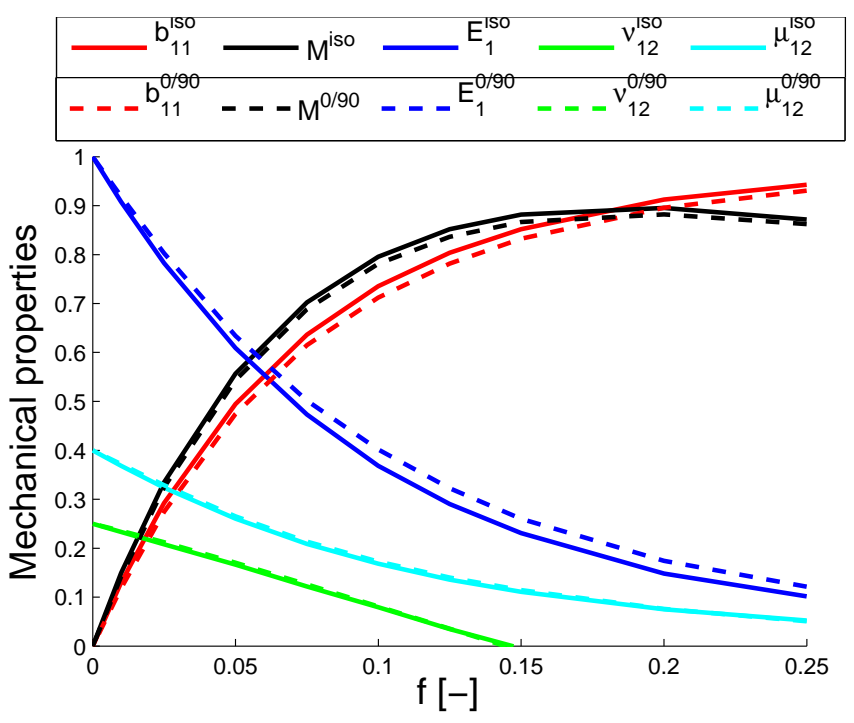

Fig. 12 Cracks of aspect ratio $1 / 10$, split in two families of orientations 0 and $\pi / 2$, or isotropically oriented.

In this situation, the results obtained with the FRIDD estimate are very good for all the poromechanical coefficients, as we can see on Figs. 13 and 14, except for the Poisson's ratio. The difficulty of estimating the Poisson's ratio is also illustrated by Segurado and Llorca [13]. However, one can argue 
that the precise value of a Poisson's ratio is of little importance when the stiffness is close to zero, as it is the case at large volume fractions of cracks.
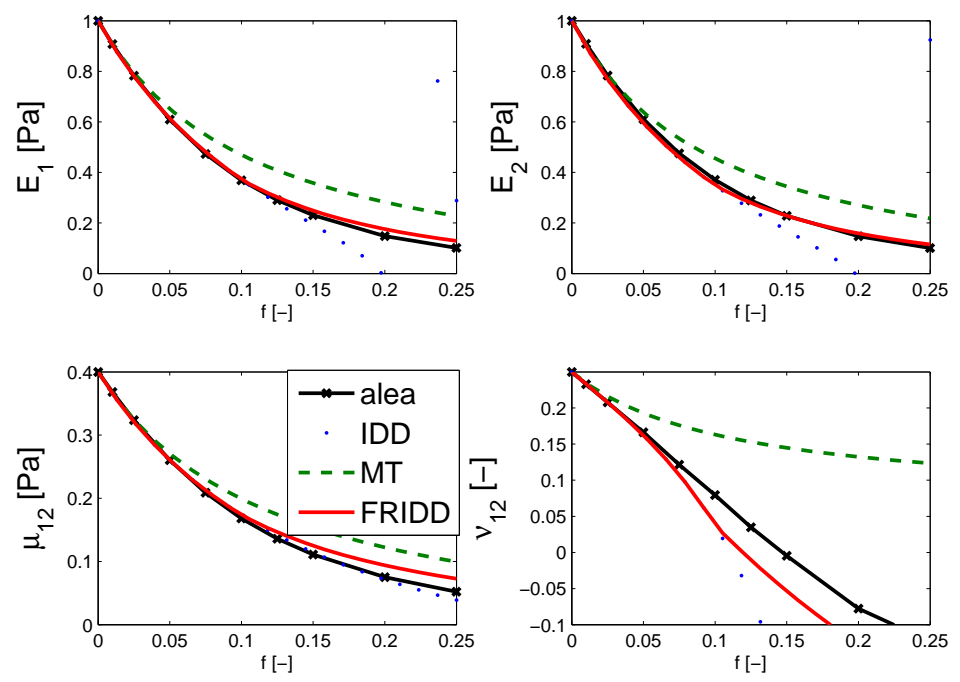

Fig. 13 Cracks of aspect ratio $1 / 10$, of isotropically distributed orientations, or in two families at 0 and $\pi / 2$. Simulations, IDD estimate with circular atmosphere, MT estimate, FRIDD estimate with evolving atmosphere. Elastic properties. Note that $\mathrm{f}=0.25$ corresponds to a crack density of $\epsilon=0.60$.
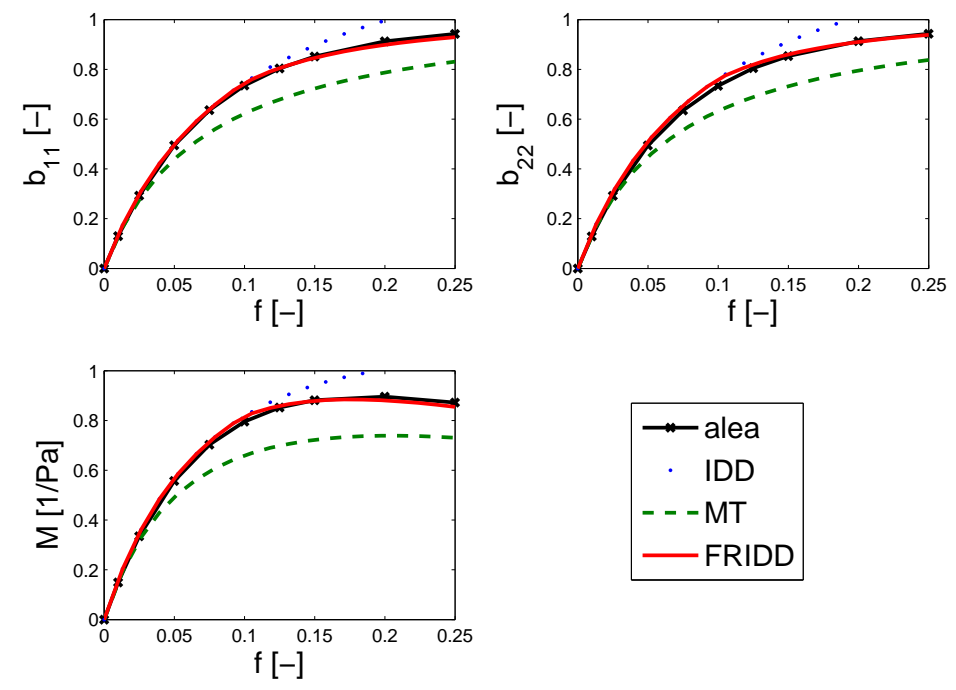

Fig. 14 Cracks of aspect ratio $1 / 10$, of isotropically distributed orientations, or in two families at 0 and $\pi / 2$. Simulations, IDD estimate with circular atmosphere, $M T$ estimate, FRIDD estimate with evolving atmosphere. Poroelastic properties. Elastic properties. Note that $\mathrm{f}=0.25$ corresponds to a crack density of $\epsilon=0.60$.

Let us comment the error made on the empirical averages of these quantities. We have plot, for 3 different sizes of voids $A$ (which is the great semi axis of an ellipse of aspect ratio 0.1) compared to domain of size $L=1$, and a constant volume fraction of voids $f=0.25$, the evolution of the empirical average and the standard deviation, as explained in $\S 5.1$. We only plot the case of periodic boundary 
conditions. As can be seen on Fig. 15, the poromechanical properties are not fully converged when the domain size is 80 times larger than the larger dimension of the void. The rate of convergence seems similar for all quantities. However, the error made on the estimation of the empirical average, given by the error bars, is not the same for all quantities. It seems that very large domains are necessary to obtain converged values of the overall properties. It might be a drawback of the fact that there is always a zone close to the external boundary where the void density is lower, due to a wall effect. Even if the size of this zone decreases with decreasing particle size, its presence might affect the rate of convergence of the homogenized properties.
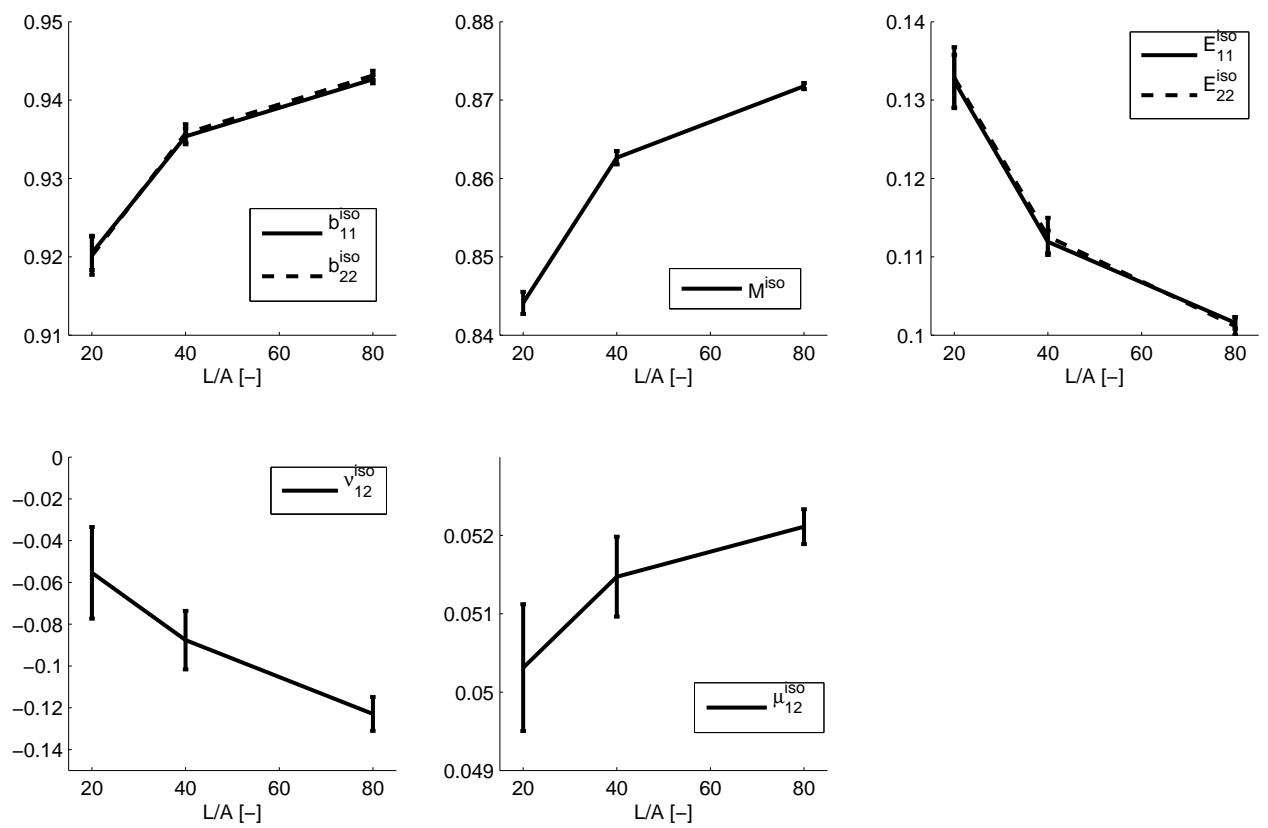

Fig. 15 Cracks of aspect ratio $1 / 10$, of isotropically distributed orientations, volume fraction $f=0.25$. Study of the convergence of the poromechanical properties determined by finite element simulations.

7.5 Discussion on the comparison of the estimates to the simulations

Our main conclusion is that the modified version of the IDD estimate, which was originally proposed by the authors under the name Full Range IDD, provide a good estimate of the overall poromechanical properties, when compared to simulations of $2 d$ microstructures generated by an algorithm of Random Sequential Addition, that is in the case of matrix/inclusion morphologies. The estimate is very good in the case of randomly oriented cracks (Figs. 13 and 14) for all poromechanical coefficients except for the Poisson's ratio. It also performs well in the case of aligned cracks (Figs. 8 and 9), but it is less accurate, particularly concerning the Young's modulus and Biot coefficient in the direction normal to the cracks.

A side conclusion of our work is the very close results obtained when estimating the mechanical properties of cracked media containing isotropically oriented cracks on the one hand, and cracks distributed in two orthogonal families on the other hand. 


\section{Conclusion}

We have built a finite element model in $2 d$ which allows computing the overall properties of porous solids with various sorts of voids, shaped as discs, or flat ellipses. Our objective was to compare the results of these computations to classical micromechanics estimates, mainly Mori-Tanaka's and the Interaction Direct Derivative estimate of Zheng and Du, but also in some cases the Self-Consistent estimate and Generalized Self Consistent estimate. We have shown that the modified version of the IDD estimate, called Full Range IDD estimate, gives very good results in all the tested cases. This conclusion is of course restricted to the case of microstructures generated with a Random Sequential Addition Algorithm which, by forbidding the interpenetration of inclusion, creates structures which clearly have matrix-inclusion morphologies. The estimates where tested up to volume fractions as large as was available using the $R S A$ algorithm with monodisperse voids (1 in the case of aligned cracks and 0.60 in the case of isotropically oriented cracks). Of course, to extend the validity of the estimates, one should test larger volume fractions by mixing various sizes of inclusions, as well as various sorts. Nevertheless, we think that our simple computations clearly demonstrate the efficiency of the Full Range IDD estimate for cracked media in $2 d$.

Therefore, we see our results as complementary to Shen and Li [7], since even if on some situations the estimates they uses might be more precise, the $I D D$ estimate from Zheng and Du we chose to use here is very versatile. In our opinion it is an important property that makes it easier to use.

\section{Acknowledgements}

This work was funded by the "Chaire Durabilité des Matériaux et des Structures pour l'Énergie" at ENPC.

\section{References}

1. Eshelby, J.D.: The elastic field outside an ellipsoidal inclusion. Proc. R. Soc. Lon. A 252(1271), 561-569 (1959)

2. Hill, R.: A self-consistent mechanics of composite materials. J. Mech. Phys. Solids 13(4), 213-222 (1965)

3. Norris, A.N.: A differential scheme for the effective moduli of composites. Mech. Mater. 4(1), 1-16 (1985)

4. Benveniste, Y.: A new approach to the application of Mori-Tanaka's theory in composite materials. Mech. Mater. 6, 147-157 (1987)

5. Hori, M., Nemat-Nasser, S.: Double-inclusion model and overall moduli of multi-phase composites. Mech. Mater. 14, 189-206 (1993)

6. Christensen, R.M., Lo, K.H.: Solutions for effective shear properties in three phase sphere and cylinder models. J. Mech. Phys. Solids 27(4), 315-330 (1979)

7. Shen, L., Li, J.: A numerical simulation for effective elastic moduli of plates with various distributions and sizes of cracks. Int. J. Solids Struct. 41(26), 7471-7492 (2004)

8. Shige, Z., Tzuchiang, W.: Effective elastic moduli of inhomogeneous solids by embedded cell model. Acta Mech. Sin. Engl. Ser. 15(4), 334-343 (1999)

9. Ponte Castañeda, P., Willis., J.R.: The effect of spatial distribution on the effective behavior of composite materials and cracked media. J. Mech. Phys. Solids 43(12), 1919-1951 (1995)

10. Hashin, Z., Shtrikman, S.: A variational approach to the theory of the elastic behaviour of multiphase materials. J. Mech. Phys. Solids 11(2), 127-140 (1963)

11. Zheng, Q.-S. and Du, D.-X.: An explicit and universally applicable estimate for the effective properties of multiphase composites which accounts for inclusion distribution. J. Mech. Phys. Solids 49, 2765-2788 (2001)

12. Miled, K., Sab, K., Le Roy, R.: Effective elastic properties of porous materials: Homogenization schemes vs experimental data. Mech. Res. Comm. 38(2), 131-135 (2011)

13. Segurado, J., Llorca, J.: A numerical approximation to the elastic properties of sphere-reinforced composites. J. Mech. Phys. Solids 50(10), 2107-2121 (2002)

14. Klusemann, B., Svendsen, B.: Homogenization methods for multi-phase elastic composites: Comparisons and benchmarks. Tech. Mech. 30(4), 374-386 (2010)

15. Huang, Y., Chandra, A., Jiang, Z.Q., Wei, X., Hu, K.X.: The numerical calculation of two-dimensional effective moduli for microcracked solids. Int. J. Solids Struct. 33(11), 1575-1586 (1996)

16. Torquato, S.: Morphology and effective properties of disordered heterogeneous media. Int. J. Solids Struct. 35(19), 2385-2406 (1998)

17. Coussy, O.: Poromechanics. Wiley (2004)

18. Charpin, L., Ehrlacher, A.: A computational linear elastic fracture mechanics-based model for alkali-silica reaction. Cem. Concr. Res. 42(4), 613-625 (2012) 
19. Charpin, L.: Modèle micromécanique pour l'étude de la réaction alcali-silice (in French). PhD thesis, Université Paris Est, France (2013)

20. FreeFem++. http://www.freefem.org/ff ++ (2013)

21. Bonnet, M., Frangi, A.: Analyse des solides déformables par la méthode des éléments finis (in French). Les éditions de l'École Polytechnique (2006)

22. Dormieux, L., Kondo, D., Ulm, F.-J.: Microporomechanics. Wiley (2006)

23. Nemat-Nasser, S., Hori, M.: Micromechanics: overall properties of heterogeneous materials. North Holland (1993)

24. Franciosi, P., Lebail, H.: Anisotropy features of phase and particle spatial pair distributions in various matrix/inclusions structures. Acta Mater. 52(10), 3161-3172 (2004)

25. Hori, M., Nemat-Nasser, S.: On two micromechanics theories for determining micro-macro relations in heterogeneous solids. Mech. Mater. 31(10), 667-682 (1999)

26. Gusev, A.A.: Representative volume element size for elastic composites: A numerical study. J. Mech. Phys. Solids 45(9), 1449-1459 (1997)

27. Anantharaman, A., Costaouec, R., Le Bris, C., Legoll, F., Thomines, F.: Introduction to numerical stochastic homogenization and the related computational challenges: some recent developments. In Weizhu Bao and Qiang Du, editors, Multiscale modeling and analysis for materials simulation, Lecture Notes Series, Institute for Mathematical Sciences, National University of Singapore 22, 197-271 (2011)

28. Kanit, T., Forest, S., Galliet, I., Mounoury, V., JeulinD.: Determination of the size of the representative volume element for random composites: statistical and numerical approach. Int. J. Solids Struct. 40, 3647-3679 (2003)

29. Mori, T., Tanaka, K.: Average stress in matrix and average elastic energy of materials with misfitting inclusions. Acta Metallurgica 21, 571-574 (1973)

30. Weng, G.J: The theoretical connections between Mori-Tanaka's theory and the Hashin-Shtrikman-Walpole bounds. Int. J. Engng Sci. 228(11), 1111-1120 (1990)

31. Zhao, Y.H., Tandon, G.P., Weng, G.J.: Elastic moduli for a class of porous materials. Acta Mech. 76, 105-130 (1989)

32. Budiansky, B., O’Connell, R.J.: Elastic moduli of a cracked solid. Int. J. Solids Struct. 12(2), 81-97 (1976)

33. Weng, G.J.: A dynamical theory for the Mori-Tanaka and Ponte Castañeda-Willis estimates. Mech. Mater. 42, 886-893 (2010)

34. Hu, G.K., Weng, G.J.: Some reflections on the Mori-Tanaka and Ponte Castañeda-Willis methods with randomly oriented ellipsoidal inclusions. Acta Mech. 140(1-2), 31-40 (2000)

35. Hu, G.K, Weng, G.J.: The connections between the double-inclusion model and the Ponte CastañedaWillis, Mori-Tanaka, and Kuster-Toksoz models. Mech. Mater. 32(8), 495-503 (2000)

36. Kanaun, S.K., Jeulin, D.: Elastic properties of hybrid composites by the effective field approach. J. Mech. Phys. Solids 49(10), 2339-2367 (2001)

37. Bornert M.: A generalized pattern-based self-consistent scheme. Comput. Mater. Sci. 5(1-3), 17-31 (1996)

38. Torquato, S.: Effective stiffness tensor of composite media: II. applications to isotropic dispersions. J. Mech. Phys. Solids 46(8), 1411-1440 (1998)

39. Ma, L., Wang, X., Feng, X.-Q., Yu, S.-W.: Numerical analysis of interaction and coalescence of numerous microcracks. Eng. Fract. Mech. 72(12), 1841-1865 (2005)

40. Kurukuri, S.: Homogenization of Damaged Concrete Meso-structures using Representative Volume Elements - Implementation and Application to SLang. Masters thesis, University of Weimar, Germany (2005)

41. Yin, H.P., Ehrlacher, A.: Size and density influence on overall moduli of finite media with cracks. Mech. Mater. 23(4), 287-294 (1996)

42. Bary, B.: Estimation of poromechanical and thermal conductivity properties of unsaturated isotropically microcracked cement pastes. Int. J. Numer. Anal. Met. 35(14), 1560-1586 (2011)

43. Stora, E., He, Q.-C., Bary, B.: Influence of inclusion shapes on the effective linear elastic properties of hardened cement pastes. Cem. Concr. Res. 36(7), 1330-1344 (2006) 